\title{
Ultrasound Biomicroscopy as a Probe of Cellular Ultrastructure
}

\author{
Yew Meng Heng (1), Matt Butler (2), Michael C. Kolios (2,3) and Gregory J. Czarnota (3,4) \\ 1 Department of Paediatric Laboratory Medicine, Division of Pathology, Hospital for Sick \\ Children, 555 University Avenue, Toronto, Ontario Canada M5G 1X8 \\ 2 University of Toronto Medical Programme and Department of Medical Biophysics, University \\ of Toronto and Ontario Cancer Institute, 610 University Avenue, Toronto, Ontario Canada \\ M5G 2M9 \\ 3 Department of Biology, Chemistry, and Chemical Engineering, and Department of \\ Mathematics, Physics and Computer Science, Ryerson Polytechnic University, 350 Victoria \\ Street, Toronto, Ontario, Canada M5B 2K3 \\ 4 Department of Radiation Oncology, Princess Margaret Hospital, 610 University Avenue \\ Toronto, Ontario Canada M5G 2M9
}

We have previously demonstrated that high-frequency ultrasound imaging at $30-50 \mathrm{MHz}$ may be used to detect apoptosis in vitro, in situ, and in vivo (1). Our previous studies pointed to changes in chromatin morphology as being critical to the ultrasound signal changes characteristic of apoptotic cells. In this study we have begun to discriminate which various subcellular components are important in the formation of ultrasound signals. Light andelectron microscopy were used for the correlative study.

Human acute myeloid leukemia cells were used for investigations of chromatin structure and its effects on ultrasound backscatter. Leukemia cells were placed in a variety of ionic environments ranging from 1/16th isotonic to 16-fold higher than isotonic ionic strength and investigated after centrifugation. A non-linear response in ultrasound backscatter signal was demonstrated that correlated with well characterized changes in chromatin structure. Aschromatin became more condensed ultrasound backscatter was found to increase up to 16-fold in intensity and when histone disassociation occurred at the highest ionic environments backscatter decreased.

Other biochemical manipulations that altered chromatin structure were also invesitigated. Cells and nuclei were digested with DNAase resulting in decreases in ultrasound backscatter. Additionally, AML cells were grown in the presence of sodium butyrate which caused the accumulation of histone acetylation. Both processes resulted in two to four-fold decreases in ultrasound backscatter (Figure 1). Rabbit red blood cells were also used to investigate the effects of the cell=s nucleus on ultrasound backscatter. Erythroblasts were isolated and compared to erythroblasts which had just extravasated their nuclei. The latter were found to have a significant decrease in ultrasound backscatter.

Other studies have also been carried out using red blood cells to investigate the effects of the cell $=\mathrm{s}$ membrane on backscatter signals. By changing the size of the membrane using different salt concentrations and its shape by using different $\mathrm{pH}$ environments we have demonstrated a non-linear effect that these parameters have on ultrasound backscatter. Stomatocytic redblood cells were found to have increased backscatter compared to regular erythrocytes. Echinoctyic cells which were spiculated had even higher backscatter levels. 
In summary, we have demonstrated in this study that both the cell's nucleus and its membrane can have important effects on ultrasound backscatter. Moreover, chromatin substructure also plays and important role in backscatter of ultrasound as do subtle membrane effects associated with changes in cell morphology. Both parameters are important in the use ofultrasound backscatter in the assessment of cell and tissue apoptosis.
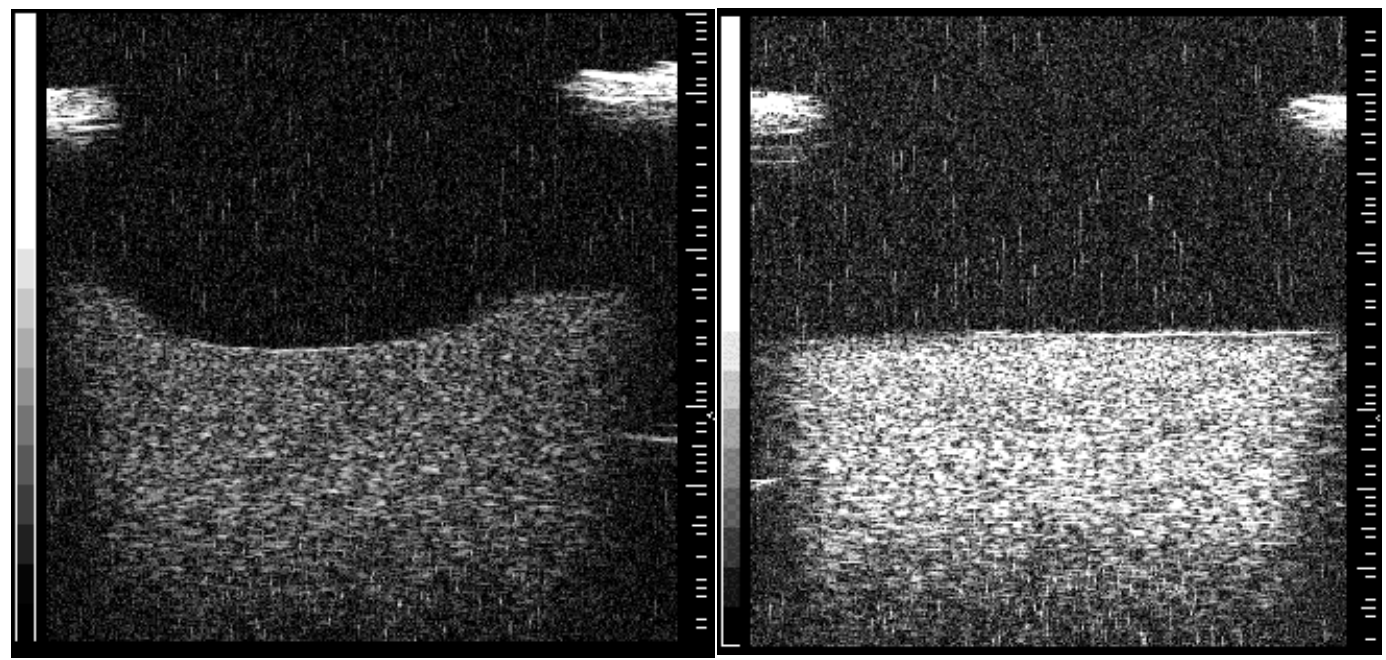

Figure 1. Left panel shows an ultrasonogram of a centrifuged sample of AML cells treated with sodium butyrate during cell growth. The panel on the right is a sample of AML cellsnot treated with the agent. Samples are immersed in isotonic phosphate buffered saline. Panels are $8 \mathrm{~mm}$ by $8 \mathrm{~mm}$ in size.

References

(1) G.J. Czarnota, M. Kolios, J. Abraham, M. Portnoy, M.D. Sherar, F.P. Ottensmeyer and J.W. Hunt. "Ultrasound Imaging of Apoptosis: High resolution Non-invasive Monitoring of Programmed Cell Death in vitro, in situ, and in vivo." British Journal of Cancer 81 520-527, 1999. 